\title{
Re-analysis of the coral Acropora digitifera transcriptome reveals a complex IncRNAs- mRNAs interaction network implicated in Symbiodinium infection
}

\author{
Chen Huang, Dongliang Leng, Shixue Sun and Xiaohua Douglas Zhang ${ }^{*}$ (D)
}

\begin{abstract}
Background: Being critically important to the ecosystem, the stability of coral reefs is directly related to the marine and surrounding terrestrial environments. However, coral reefs are now undergoing massive and accelerating devastation due to bleaching. The fact that the breakdown of symbiosis between coral and the dinoflagellate, zooxanthellae, has been well elucidated as the main cause of bleaching, implying the establishment of symbiosis with zooxanthellae plays a crucial role in maintaining coral survival. However, the relevant molecular and cellular mechanisms have not been well studied yet. In this study, based on the deep RNA-sequencing data derived from Mohamed, A. R. et al., an integrated transcriptome analysis was performed to deeply investigate global transcriptome changes of the coral Acropora digitifera in response to infection by dinoflagellate of the genus Symbiodinium.

Results: The results revealed that compared to RefTranscriptome_v1.0 (A. digitifera transcriptome assembly v1.0), numerous novel transcripts and isoforms were identified, the Symbiodinium-infected coral larvae at $4 \mathrm{~h}$ generated the highest proportion of specific isoforms. Alternative splicing analysis showed that intron retention predominated in all alternative transcripts among six statuses. Additionally, 8117 IncRNAs were predicted via a stringent stepwise filtering pipeline. A complex IncRNAs-mRNAs network including 815 IncRNAs and 6395 mRNAs were established, in which 21 IncRNAs were differentially expressed at $4 \mathrm{~h}$ post infection. Functional clustering analysis for those differentially IncRNAs-coexpressed mRNAs demonstrated that several biological processes and pathways related to protein kinase activity, metabolic pathways, mitochondrion, ribosome, etc. were enriched.
\end{abstract}

Conclusions: Our study not only refined A. digitifera transcriptome via identification of novel transcripts and isoforms, but also predicted a high-confidence dataset of IncRNAs. Functional study based on the construction of IncRNAsmRNAs co-expression network has disclosed a complex IncRNA-mediated regulation in response to Symbiodinium infection exhibited in A. digitifera. Once validated, these IncRNAs could be good potential targets for treatment and prevention of bleaching in coral.

Keywords: Acropora digitifera, Symbiodinium, Deep RNA-sequencing, Transcriptome, Alternative splicing, Long noncoding RNAs

\footnotetext{
* Correspondence: douglaszhang@umac.mo

Faculty of Health Sciences, University of Macau, Taipa, Macau
}

(c) The Author(s). 2019 Open Access This article is distributed under the terms of the Creative Commons Attribution 4.0 International License (http://creativecommons.org/licenses/by/4.0/), which permits unrestricted use, distribution, and reproduction in any medium, provided you give appropriate credit to the original author(s) and the source, provide a link to the Creative Commons license, and indicate if changes were made. The Creative Commons Public Domain Dedication waiver (http://creativecommons.org/publicdomain/zero/1.0/) applies to the data made available in this article, unless otherwise stated. 


\section{Background}

Coral reefs, also known as "rainforests of the sea", are one of the most important ecosystems on the planet. Although only covering less than $0.1 \%$ of the surface of the world's ocean, they can support more than one-quarter of all marine organisms [1]. Thus, coral reefs play a vital role in the marine nitrogen cycle upon which other marine organisms depend. As an important component in marine ecosystems, the healthy, abundant and diverse coral reefs with residing marine organisms have direct influence on the surrounding terrestrial and marine environments. However, coral reefs and associated tropical nearshore ecosystems have suffered from massive, long-term decline in abundance, diversity, as well as habitat structure since early twentieth century due to the widespread outbreaks of coral disease and bleaching $[2,3]$. There are multiple factors resulting in coral bleaching, which can be divided into two main categories: environmental factors and anthropogenic factors [4, 5]. For example, environmentally destructive activities of human, like overfishing and seawater pollution lead to oxygen starvation, ocean acidification [6] and change in chemistry in seawater [7]. On the other hand, global warming is an important environmental factor which leads to deterioration of coral reef ecosystems $[8,9]$. It is well known that bleaching has been attributed to the dissociation of the symbiotic relationship between algae and their hosts, and all these factors would disrupt the balance of symbiotic relationship between host coral and their symbionts, i.e., microscopic algae of the genus Symbiodinium, commonly referred to as zooxanthellae. Zooxanthellae are single-celled dinoflagellates that are capable of inhibiting symbiosis with corals. They are photosynthetic organisms, which provide their host with the organic carbon products of photosynthesis for host metabolism, growth and reproduction. In exchange, the host will supply nutrients, carbon dioxide, and habitat with access to sunshine for zooxanthellae. However, when the zooxanthellae are exposed under stress, such as increased water temperature, oxygen starvation, or even bacterial infection, they will die or leave their host - a process known as bleaching $[10,11]$.

Due to the crucial roles of coral in maintaining marine ecosystems, continuous investigation on the mechanism of coral bleaching has been carried out since the end of twentieth century $[12,13]$. Despite diverse external and internal factors that drive coral bleaching have already been clarified, the molecular mechanisms underlying the establishment, maintenance or collapse when bleaching occurs are still poorly understood. Thus, deep investigation of the relevant molecular mechanisms of coral bleaching, and how the coral responses to bleaching in molecular level should have great significance in prevention and therapy of coral bleaching. Fortunately, advancement of high throughput sequencing (HTS) technologies in recent decades such as next generation sequencing (NGS) enables us to investigate the genetic and epigenetic molecular bases underlying coral health and disease. Chuya Shinzato et al. [14] firstly published the whole genome of coral Acropora digitifera utilizing Roche 454 GS-FLX and Illumina Genome Analyser IIx (GAIIx) sequencing platforms to investigate the molecular basis of symbiosis and responses to environmental changes. They reported that $A$. digitifera seemed to lack an enzyme essential for cysteine biosynthesis, implying this coral may rely on its symbionts for cysteine. DeSalvo, M. K. et al. [15] used DNA microarray technique to study gene expression changes during thermal stress and bleaching in the Caribbean coral Montastraea faveolata. They found that some genes involved in oxidative stress, $\mathrm{Ca}^{2+}$ homeostasis, cell death, protein synthesis, etc. could be affected when the coral suffered from thermal stress and bleaching. In addition, the transcriptomics analysis of Pinzon J. H. et al. [16] revealed that the immune-related genes would be changed during and after bleaching in coral $O$. faveolata. Notably, Mohamed, A. R. et al. [17] designed three different time points ( $4 \mathrm{~h}, 12 \mathrm{~h}$ and $48 \mathrm{~h}$ ) to compare the gene expression of $A$. digitifera between normal samples and Symbiodinium-infected samples using Illumina RNA-Seq technology. They found that a small number of genes were differentially expressed at $4 \mathrm{~h}$ post-infection but returned to baseline level at $12 \mathrm{~h}$ and $48 \mathrm{~h}$, implying the transcriptome resilience of $A$. digitifera in response to the change of external environment. However, most of the genomic and transcriptomic studies about coral bleaching focuses on the protein-coding genes, such as gene expression or transcriptional changes of coral in response to bleaching. Fewer if any studies explore the significance of non-coding sequences, i.e., long non-coding RNAs (lncRNAs). LncRNAs are defined as non-protein-coding transcripts longer than 200 nucleotides [18]. Despite they were initially regarded as "junk genomic sequences", increasing studies reveal that lncRNAs exhibit diverse regulatory or epigenetic functions [19], and are proven to be correlated to many diseases, particularly cancers [20-23]. Accumulating evidences demonstrated that lncRNAs may serve as crucial transcriptional regulator of layers involved in a diversity of biological processes and pathways [24, 25]. In addition, IncRNAs can interact with RNAs, DNAs, and proteins to form a complex or even triplex to exert their various functions, such as suppressing or enhancing the expression of genes, activating or inactivating the transcription of genes, etc. [25]. Because of the important roles in diverse biological regulatory cascades and pathways, lncRNAs have emerged as a research hotspot in human disease. Despite the majority of lncRNA studies focuses on human, 
the number of studies about lncRNAs of other organisms is growing. For examples, NONCODE database has recruited numerous lncRNAs derived from diverse model organisms, such as mouse, cow, rat, chimpanzee, and yeast [26]. Furthermore, some studies tried to investigate lncRNAs from simpler organisms. Notably, Gaiti Federico et al. [27] identified a large number of lncRNAs in the demosponge Amphimedon queenslandica, a morphologically simple, early-branched metazoan, and revealed that regulation of lncRNAs existed in the development of ancient metazoans, suggesting that lncRNAs are essential regulatory elements regardless of species complexity. Therefore, bleaching, also known as "disease" in coral, which has been proven to be correlated to the expressional change of many genes, such as immune-related genes, thermal-related genes, should be associated to the regulations of lncRNAs.

Similar to mRNAs in many ways, e.g., both lncRNAs and mRNAs are generated by the same histone modification, transcribed by the same RNA polymerase II and could be polyadenylated, most lncRNAs could be retrieved from RNA-seq based on the RNA 'Poly (A)' library $[28,29]$. Indeed, our previous study [30] about IncRNA of coral successfully identified numerous IncRNAs based on the transcriptome datasets of two corals species: Protopalythoa varibilis and Palythoa caribaeorum. In addition, we predicted possible target mRNAs for a few lncRNAs exhibiting ultra-conserved regions and revealed a post-transcriptional pattern existed in ancient cnidarian animals. We discussed their possible associations in bleaching via comparison of healthy coral with the colony undergoing bleaching. In our previous study there are limitations. For example, the coral we investigated had no genome reference, which would result in massive false positive results during lncRNAs prediction. Differential expression analysis of lncRNAs involved in bleaching lacked the sufficient statistics power to support our hypothesis with only two coral samples (one healthy coral and one colony undergoing bleaching). Therefore, in the present study, on the basis of RNA sequencing dataset of $A$. digitifera from Mohamed, A. R. et al's study [17], an integrated transcriptome analysis was conducted, aiming at further investigating the transcriptomic changes (including mRNAs and lncRNAs) of $A$. digitifera in response to Symbiodinium infection, on the basis of expression level as well as sequence structure, and discovering the possible roles of IncRNAs involved in Symbiodinium infection. Our study not only disclosed, for the first time, a high-confidence dataset of lncRNAs for $A$. digitifera, but also revealed a complex interaction between mRNAs and lncRNAs in A. digitifera, as well as their possible roles in Symbiodinium infection. We believe our study can well complement Mohamed, A. R. et al's study and provide new insights for the study of molecular base of coral bleaching.

\section{Results}

Raw data processing and transcriptome identification

To identify as many transcripts (including the lncRNAs) as possible from $A$. digitifera, we proposed a systemic bioinformatics pipeline (Fig. 1). First, each library (200 bps pair-end) was sequenced on Illumina HiSeq 2000 platform respectively, yielding a total of 704,650,768 reads, and quality filtering was performed using Trimmomatic to obtain $659,941,956$ clean reads, which are composed of $65,048,863,068$ bases (Additional file 1: Table S1). Then the filtered reads for each library were aligned against $A$. digitifera genome sequence using HISAT2. The result indicated that the majority of them (range from 75\% 78\%) could be mapped to A. digitifera genome properly (Additional file 2: Table S2). Afterwards, successfully aligned reads were assembled into 59,904 transcripts using StringTie (Table 1, Additional file 3: Table S3, Additional file 4: Figure S1). Additionally, all the clean data were compared to the assembled transcripts using Bowtie 2 to assess the quality of those assembled transcripts. The results showed that majority of the reads $(\sim 80 \%)$ could be well mapped into the assembled transcripts (Additional file 5: Table S4). Basic coverage statistics of the clean reads mapped into the assembled transcripts achieved by QualiMap [31] indicated that majority of transcripts (over 95\%) could be well covered by the clean reads (Additional file 6: Figure S2), which demonstrated that our analysis yielded a high-quality dataset of transcripts.

\section{Statuses-specific isoforms and alternative splicing modes} We next compared the assembled transcripts of coral larvae in six different status (control in $4 \mathrm{~h}, 12 \mathrm{~h}$ and 48 h, Symbiodinium-infected in $4 \mathrm{~h}, 12 \mathrm{~h}$ and $48 \mathrm{~h}$ ). The result indicated that, of six statuses, Symbiodinium-infected at $4 \mathrm{~h}$ had the highest proportion of specific transcripts $(45,319,75.6 \%)$, followed by Symbiodiniu$m$-infected at $48 \mathrm{~h}(44,395,74.1 \%)$ and control at $4 \mathrm{~h}$ (43,762, 73.1\%), whereas Symbiodinium-infected at $12 \mathrm{~h}$ $(42,827,71.5 \%)$ had the lowest proportion (Fig. 2a). Comparison of novel status-specific transcripts generated from both known and novel transcripts demonstrated similar patterns (Fig. 2b). Furthermore, we utilized AStalavista [32] to detect the five important alternative splicing modes from the assembled transcripts for each status, namely intron retention, exon skipping, alternative $3^{\prime}$-acceptor, alternative $5^{\prime}$-donor and mutually exclusive exon (Fig. 2c). For all transcripts of six status, intron retention predominated, accounting for around $25 \%$ of alternative transcripts (Fig. 2d). Intron retention, which is one of most important alternative splicing mode, could introduce stop codons and change open reading frames (ORFs), thereby causes functional mutations [33, 34]. Splicing mode was not uniform across the 


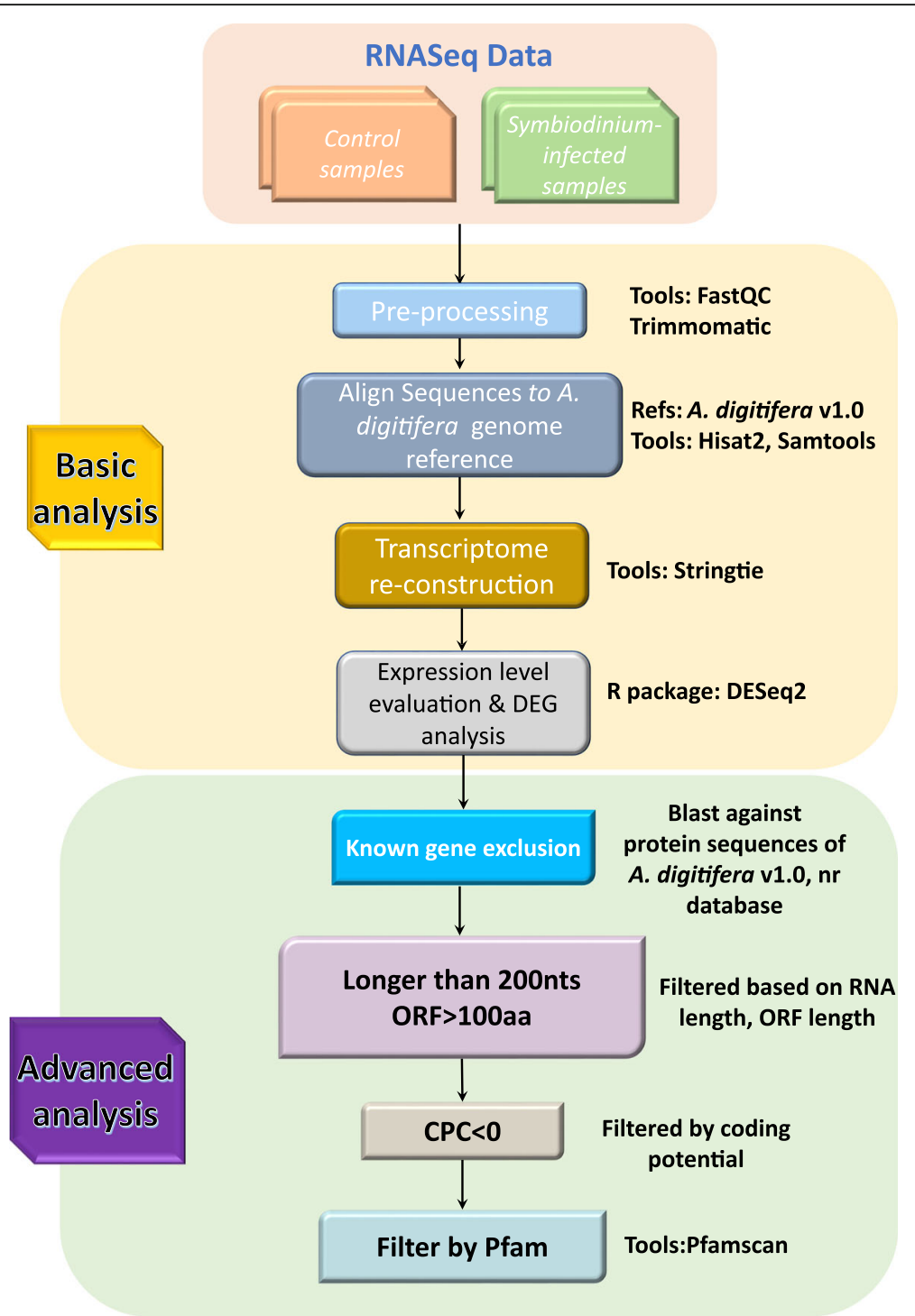

Fig. 1 The bioinformatics pipeline used for the identification of transcripts and IncRNAs of the coral A. digitifera

six statuses. For instance, mutually exclusive exon in which either of two exons was used, contributed to the most of alternative transcripts in control $12 \mathrm{~h}$ and $48 \mathrm{~h}$ and Symbiodinium-infected $12 \mathrm{~h}$ and $48 \mathrm{~h}$ (Fig. 2d).

\section{Comparison analysis reveals novel isoforms}

We compared the assembled transcripts against the $A$. digitifera transcriptome assembly v1.0 (RefTranscriptome_v1.0, 36,780 transcripts) using BLASTn with cut-off E-value 1e-5. The result showed that most of the assembled transcripts $(51,347)$ exhibited high homology to at least one transcript of RefTranscriptome_v1.0. More importantly, 7814 novel transcripts were identified in our analysis. For example, the transcript MSTRG.26111.1 which contained five isoforms (annotated as protein ATP-binding cassette sub-family A member 5 in
Swiss-Prot database) was found but missing in Reftranscriptome_v1.0 (Fig. 3a). Moreover, compared to Reftranscriptome_v1.0, our transcriptome data revealed several novel isoforms. For instance, four isoforms of MSTRG.53.1 (annotated as protein Trafficking protein particle complex subunit 1 in Swiss-Prot database) were detected: one isoform in control $04 \mathrm{~h}$ shared a similar structure with the reported annotation and the other three were novel (control $12 \mathrm{~h}$, infected $12 \mathrm{~h}$ and infected 48 shared the same isoform) (Fig. 3b).

\section{Transcriptome quantification and differential expression analysis}

In addition to comparison of transcripts in sequence structures, we also compared the expression level of those transcripts in six statuses. We quantified the 
Table 1 The basic statistics of assembly of the coral A. digitifera transcriptome

\begin{tabular}{|c|c|c|c|c|}
\hline Sample ID & Status & Individual transcripts & Merged transcripts & Total transcripts \\
\hline SRR3106384 & control_04h_rep1 & 38,019 & 38,697 & 59,904 \\
\hline SRR3106385 & control_04h_rep2 & 37,977 & & \\
\hline SRR3106386 & control_04h_rep3 & 37,633 & & \\
\hline SRR3106387 & Symbiodinium_infected_04h_rep1 & 44,484 & 44,889 & \\
\hline SRR3106388 & Symbiodinium_infected_04h_rep2 & 46,656 & & \\
\hline SRR3106389 & Symbiodinium_infected_04h_rep3 & 39,467 & & \\
\hline SRR3106390 & control_12h_rep2 & 42,548 & 38,249 & \\
\hline SRR3106391 & control_12h_rep3 & 44,208 & & \\
\hline SRR3106392 & Symbiodinium_infected_12h_rep2 & 41,453 & 37,844 & \\
\hline SRR3106393 & Symbiodinium_infected_12h_rep3 & 40,321 & & \\
\hline SRR3106394 & control_48h_rep1 & 39,889 & 39,468 & \\
\hline SRR3106395 & control_48h_rep2 & 31,501 & & \\
\hline SRR3106396 & control_48h_rep3 & 43,208 & & \\
\hline SRR3106397 & Symbiodinium_infected_48h_rep1 & 46,655 & 43,466 & \\
\hline SRR3106398 & Symbiodinium_infected_48h_rep2 & 37,670 & & \\
\hline SRR3106399 & Symbiodinium_infected_48h_rep3 & 40,717 & & \\
\hline
\end{tabular}

abundance of those lncRNAs (Additional file 7: Figure S3) and mRNAs expression based on the read counts of clean reads mapped to the assembled transcripts for each library using featureCounts [35], then the read counts were normalized using $\mathrm{R}$ package DESeq2 [36] (Additional file 8: Figure S4). By principle component analysis (PCA), the clustering of all coral larvae in six statues based on the normalized read counts was visualized in Additional file 9: Figure S5. Moreover, in accordance with Mohamed A. R. et al's study [17], we performed differential expression analysis for these transcripts using DESeq2 between Symbiodinium-infected replicates and control samples at three time points: $4 \mathrm{~h}, 12 \mathrm{~h}$ and $48 \mathrm{~h}$, respectively. The results showed that 1036 (1.73\%) differentially expressed transcripts (DETs) could be detected at $4 \mathrm{~h}$ comparison of Symbiodinium-infected samples vs control samples (Mohamed A. R. et al's study identified 1073 DEGs using edgeR package), of which 630 were downand 406 were up-regulated. The range of $\log 2$ fold change and false discovery rate as well as hierarchical clustering of significant DETs at $4 \mathrm{~h}$ time point was visualized in Fig. 4. Regarding the comparison results at $12 \mathrm{~h}$ and $48 \mathrm{~h}$, as in Mohamed A. R. et al's study, no significant differentially expressed transcripts could be found at the $12 \mathrm{~h}$ and $48 \mathrm{~h}$ post-infection. Next, Gene Ontology (GO) and KEGG pathway analysis for those differentially expressed transcripts achieved by DAVID [37] detected 39 significantly overrepresented GO terms and 9 pathways which mainly involved in protein synthesis and energy metabolism (Fig. 4c). For example, the top eight terms are structural constituent of ribosome, translation, ribosome, cytosolic small ribosomal subunit, cytochrome-c oxidase activity, and small ribosomal subunit, etc. (yellow highlight in Additional file 10: File S1). Meanwhile, IPATH-pathway analysis also indicated that the majority of differentially expressed RNAs enriched several pathways of energy metabolism (Additional file 11: Figure S6). These results indeed were supported by Mohamed A. R. et al., who demonstrated that protein synthesis and oxidative metabolism were suppressed during the initial Symbiodinium infection [17]. To our best knowledge, symbionts like Symbiodinium living inside coral provide energy products through photosynthesis process. In exchange, Symbiodinium obtains carbon dioxide and ammonium from coral for photosynthesis [38]. The fact that loss of Symbiodinium would disrupt the balance of symbiotic relationship between coral and Symbiodinium has been widely accepted as the essential reason of bleaching. Our analysis revealed that some metabolic pathways, particularly energy metabolism pathways were temporarily suppressed at $4 \mathrm{~h}$ post infection. Moreover, our analysis revealed some differentially expressed transcripts were involved in the regulation of telomerase (Fig. 4c, red highlight in Additional file 10: File S1) and p38MAPK signaling cascades (orange highlight in Additional file 10: File S1). The functions of telomerase has been well characterized to be related to cell proliferation and apoptosis [39], and P38 MAPKs belongs to a conserved subfamily of MAPKs involved in the response to stress found in diverse eukaryotic cells. These findings implied that apart from protein synthesis and energy metabolism, Symbiodinium infection may also affect the function of cell differentiation, apoptosis and autophagy in coral. 


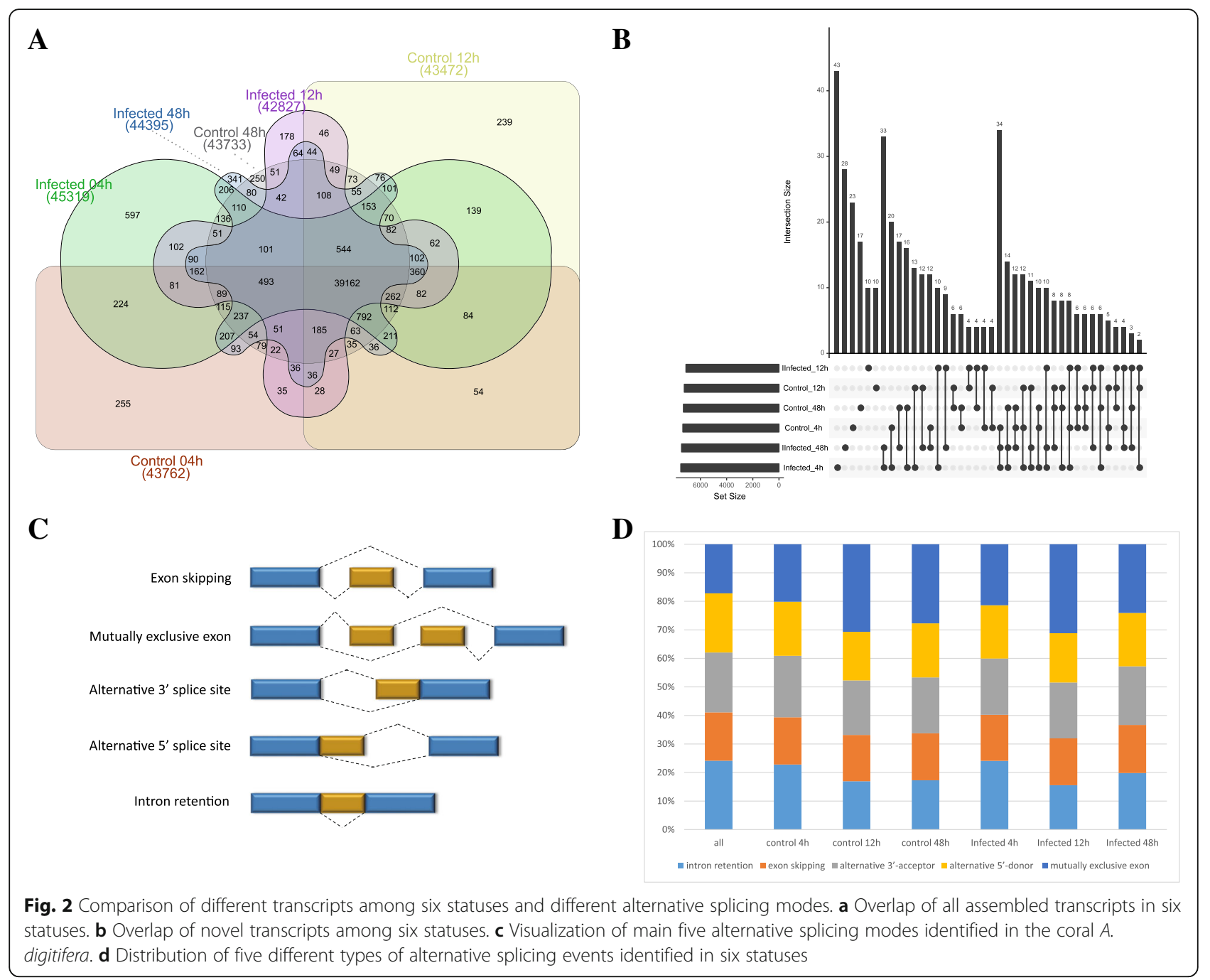

\section{Identification of IncRNAs}

In order to investigate the IncRNAs in A. digitifera, we proposed a stringent stepwise filtering pipeline to identify lncRNAs transcribed in A. digitifera (Fig. 1). Similar to our previous study on identification of lncRNAs based on the RNA-seq data of the coral Protopalythoa and Palythoa [30], the main concept of identifying lncRNAs is to remove as many potential protein-coding transcripts as possible. Specifically, a total of 50,383 transcripts that aligned to the known protein sequences of $A$. digitifera proteins dataset v1.0 (RefProtein_v1.0, 26,275 proteins), NCBI non-redundant (nr) database and Swiss-Prot database were removed. Then, the remaining transcripts shorter than 200 nucleotides were filtered out. The remaining transcripts were subjected to the ORF prediction to remove the transcripts of protein-coding potential based on the maximum ORF size of 100 amino acid residues. After these steps, 8130 lncRNA candidates were retained. CPC [40] was applied to further assess the protein-coding potential of those lncRNA candidates. Finally, the remaining transcripts were subjected to Pfam database to search for the functional domain/motif using Pfamscan. As a result, 8117 lncRNAs were obtained.

\section{Characteristics of IncRNAs}

To better understand the features of lncRNAs of $A$. digitifera, we compared the transcript length, exon counts, open reading frame (ORF) length and expression level of those 8117 lncRNAs with 50,383 mRNAs. In agreement with previous studies of IncRNAs in other eukaryotes [41-43], the length of the majority of identified lncRNAs and the corresponding ORF was much shorter than that of mRNAs (Fig. 5a, and b), and the exon count of the lncRNAs was also less than that of mRNAs (Fig. 5c). On the other hand, the comparison of expression level between lncRNAs and mRNAs showed no significant differences among the six statuses (Fig. 5d).

Furthermore, we compared the lncRNAs of $A$. digitifera with other known lncRNAs derived from NONCODE database v3.0, 411 [26] (including 411,552 


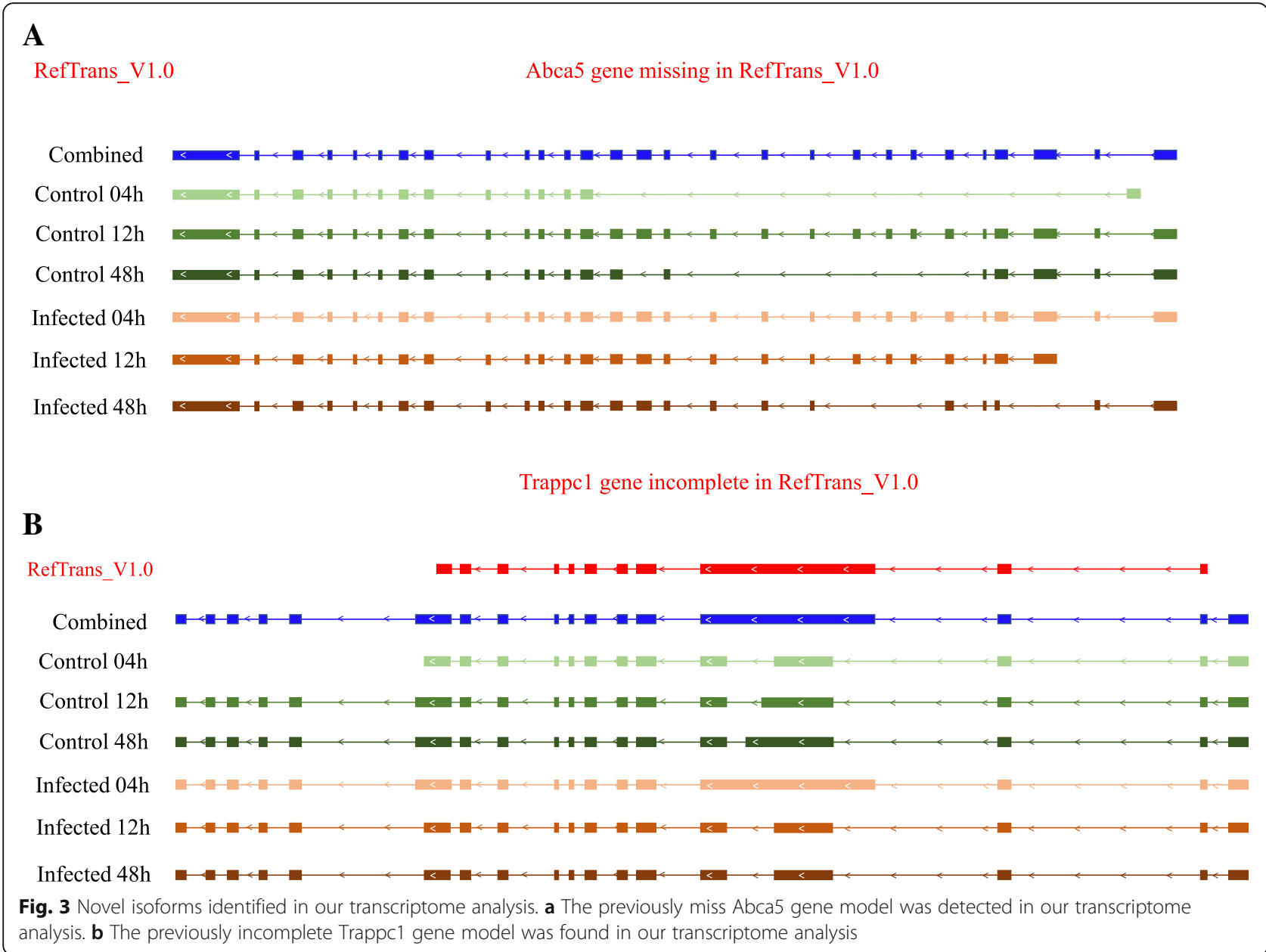

lncRNAs of diverse organisms, such as Homo sapiens, Mus musculus, Arabidopsis thaliana, etc.) using BLASTn (cutoff E-value $\leq 1 \mathrm{e}-3$ ). In agreement with our previous study of lncRNAs in coral [30], 55 lncRNAs (less than 1\%) could be compared to the known lncRNAs from NONCODE. The low percentage was expected since lncRNAs exhibit rather low conservation among a diversity of species [44-46]. However, lack of conservation does not suggest lack of function. Indeed, like our previous study on coral lncRNAs [30], we also found ultra-conserved region (UCR) among these compared lncRNAs. UCR have already been widely identified among various mammals, like human, rat, mouse, which were inferred to play important roles in function of IncRNAs [44-46]. On the other hand, those lncRNAs of A. digitifera were compared to the other predicted IncRNAs of coral: Protopalythoa and Palythoa from our previous study [30] using BLASTn (cutoff E-value $\leq 1 \mathrm{e}-5$ ). The results showed that 1059 lncRNAs could be well compared. The possible reason that a more stringent cutoff resulted in more matches could be that these IncRNAs are relatively conserved in closely-related species.
mRNAs-IncRNAs interaction network

To investigate the functions of lncRNA of $A$. digitifera, the possible mRNAs-lncRNAs interaction network was established. First, for each of 8117 lncRNAs which were obtained in previous analysis, we predicted the possible mRNA targets from the 50,383 annotated mRNAs based on the expression correlation coefficient, the mRNAlncRNA pairs which had absolute value of Pearson correlation $\geq 0.95$ and $P$-value $<0.05$ (the significance of cutoff value was validated by permutation test, detailed process and results see Methods section) were retained. As a result, a total of 23,784 mRNA-lncRNA interaction relationships were identified which involved $815 \operatorname{lncRNAs}$ and 6395 mRNAs. Additionally, those 23,784 mRNA-lncRNA pairs were subjected to RIblast to calculate the interaction power based on the free energy for hybridization (Fig. 6). Afterwards, all the target mRNAs of those lncRNAs were subjected to DAVID for Gene Ontology (GO) analysis and KEGG pathway analysis. The results indicated that 210 Gene Ontology terms and 9 KEGG pathways were significantly enriched (EASE score $<0.05$ ) (Additional file 12: File S2). These findings clearly showed that these lncRNAs 

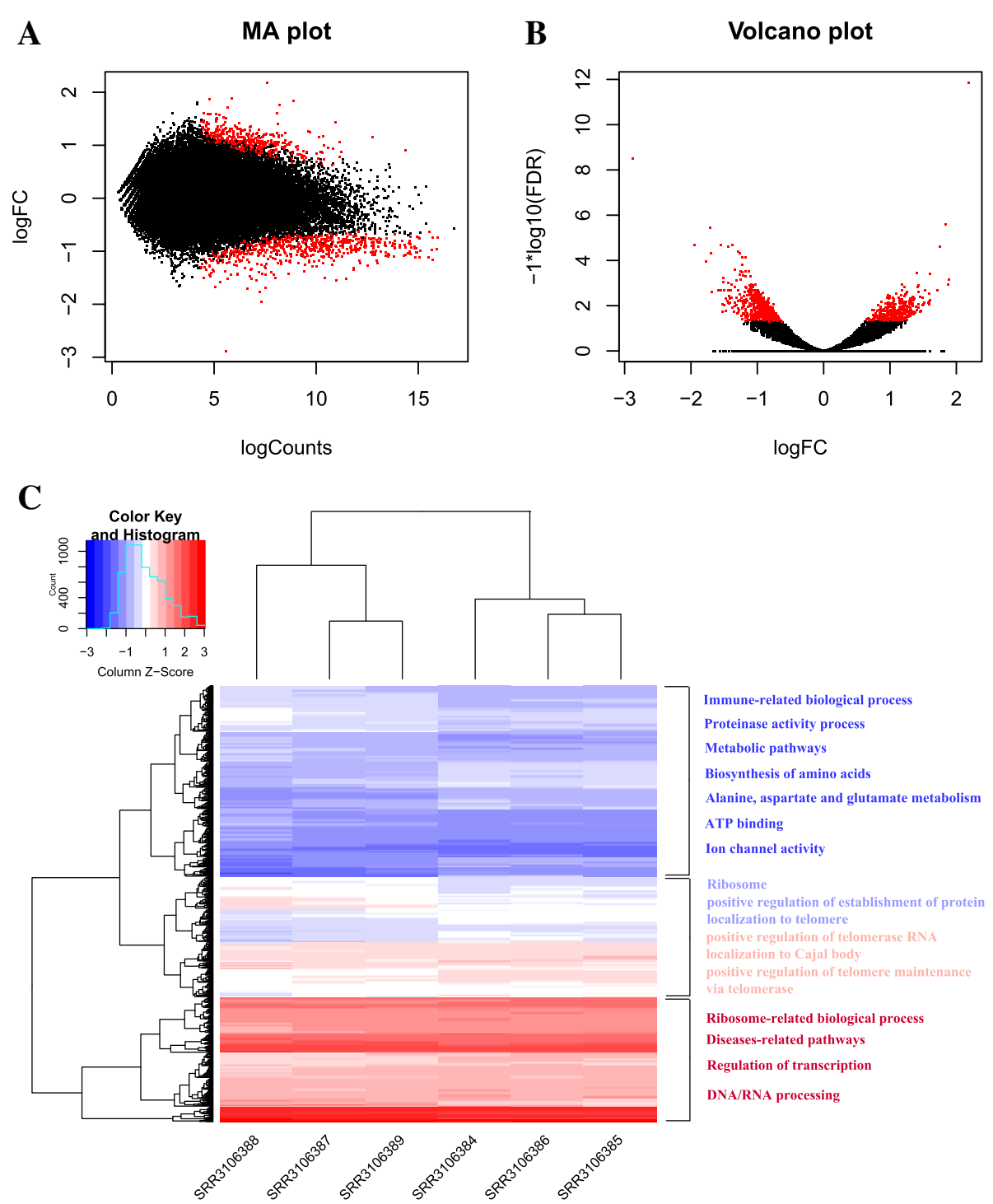

Fig. 4 Differentially expressed transcripts identified during the comparison between control samples versus Symbiodinium-infected repeats at $4 \mathrm{~h}$ post infection. a MA plot for the transcriptome expression profiles of coral A. digitifera. $\mathbf{b}$ Volcano plot for the transcriptome expression profiles of coral $A$. digitifera, showing false discovery rate (-log10FDR) as a function of $\log 2$ (fold change). The red dots represent the differentially expressed transcripts at adjusted $P \leq 0.05$. c Heatmap of differential transcriptome expression profiles. The heatmap was visualized using R package gplots, all expression values were normalized using Z-score, and the differentially expressed transcripts were initially clustered using average linkage clustering

might be involved in a diversity of biological processes and pathways, particularly protein synthesis and processing, cell adhesion via acting on their target mRNAs, i.e., protein binding, ATP binding, Golgi membrane, Golgi apparatus, cell-cell junction, focal adhesion, etc. (Additional file 12: File S2). It was well known that cell adhesion played a key role in host-Symbiodinium interactions. The initial interaction with Symbiodinium should involve cell adhesion [17]. In addition, among the mRNAs-lncRNAs interaction network, we noticed that 21 lncRNAs were differentially expressed at $4 \mathrm{~h}$ post infection. GO and KEGG pathway analysis of the co-expressed mRNAs for each differentially expressed lncRNAs revealed several biological processes and pathways that were significantly enriched, despite some lncRNAs had only a few target mRNAs (Table 2). For instance, lncRNA MSTRG.6940.1 had only five target mRNAs based on our prediction, whereas functional enrichment analysis for target mRNAs demonstrated that two ribosome-related GO categories could be significantly enriched, suggesting that it might participate the regulation of protein synthesis via acting on their target mRNAs. Intriguingly, we observed among those differential expressed lncRNAs-mRNAs relationship, a few decreased IncRNAs together with their co-expressed down-regulated-mRNAs formed a small complex sub-network (red frame in Fig. 6 and 7). 


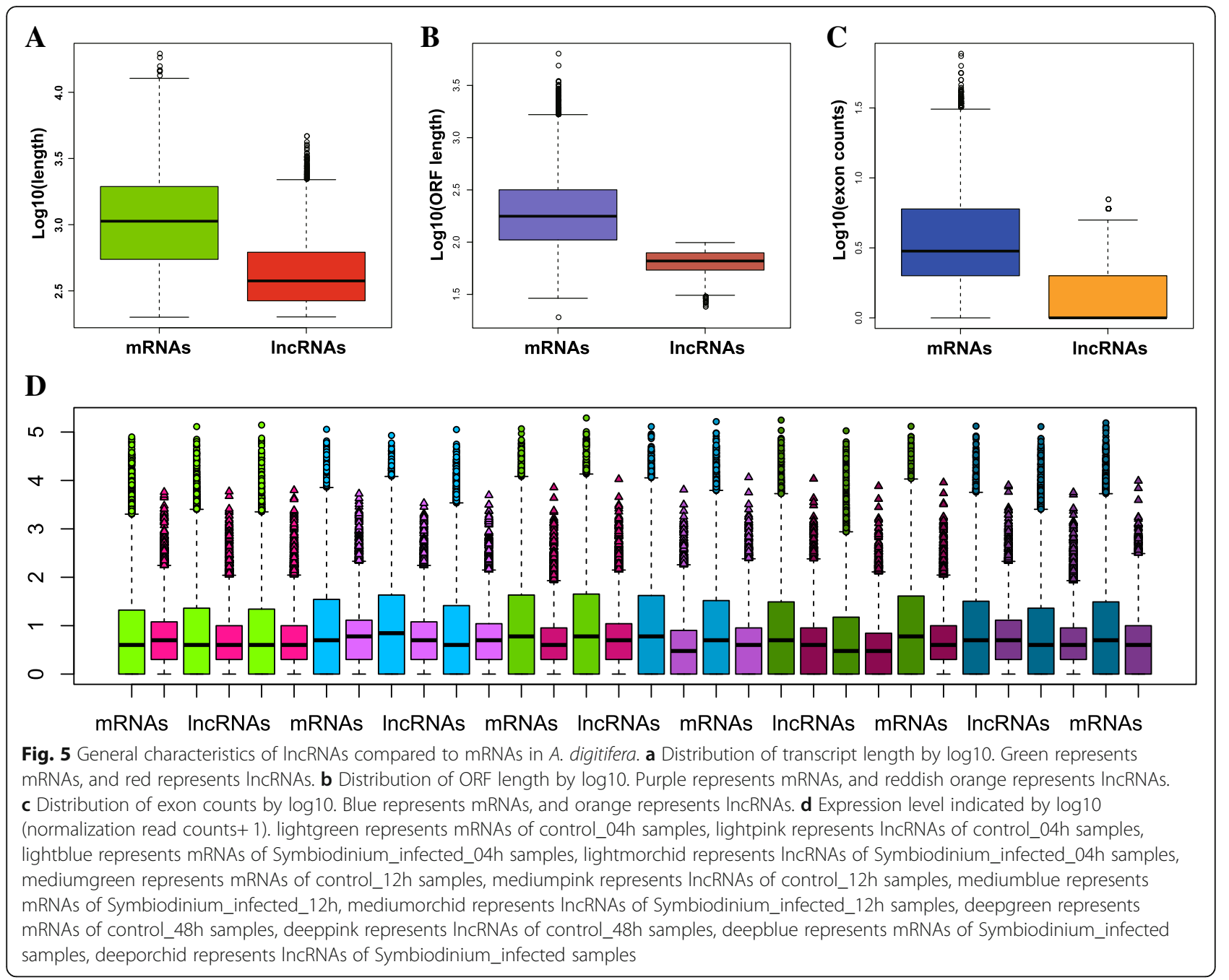

Moreover, functional analysis showed that these down-regulated mRNAs-lncRNAs interactions were involved in several biological processes and pathways related to energy metabolism, protein synthesis and cell apoptosis, including cytochrome-c oxidase activity, ribosome, proton-transporting ATPase activity, rotational mechanism, translation and mitochondrion-related process, etc. For example, suppression of cytochrome-c oxidase activity would affect the functions of chondriosome, which would further rise up to cell apoptosis [47]. Thus, our findings suggested that these lncRNAs might participate the repression of gene expression of those biological processes and pathways, like energy metabolism, cell apoptosis via acting on the relevant target mRNAs at early stage of Symbiodinium infection.

\section{Discussion}

Compared to Mohamed A. R. et al's study, despite our analysis indicated that the similar number of transcripts were differentially expressed (1036 in our study and
1073 in Mohamed A. R. et al's study), the GO and KEGG analysis showed distinct results. For instance, apart from protein synthesis and energy metabolism that had been well illustrated in Mohamed A. R. et al's study, three GO_BP categories which were enriched by significant differentially expressed transcripts at $4 \mathrm{~h}$ post Symbiodinium infection were involved in cell differentiation and apoptosis, i.e., GO:1904874 positive regulation of telomerase RNA localization to Cajal body, GO:0038066 p38MAPK cascade. It should be noted that Mohamed A. R. et al. also identified 11 DEGs involved in apoptosis functions. However, those findings were obtained by literature searches, not by direct GO functional enrichment analysis. The difference may be explained by different bioinformatic analysis strategies applied. For instance, the differential expression analysis in Mohamed A. R. et al's study was performed based on A. digitifera transcriptome assembly v1.0 which contains 36,780 transcripts, whereas in our study, on the basis of de novo assembly, a total of 59,904 were eventually 


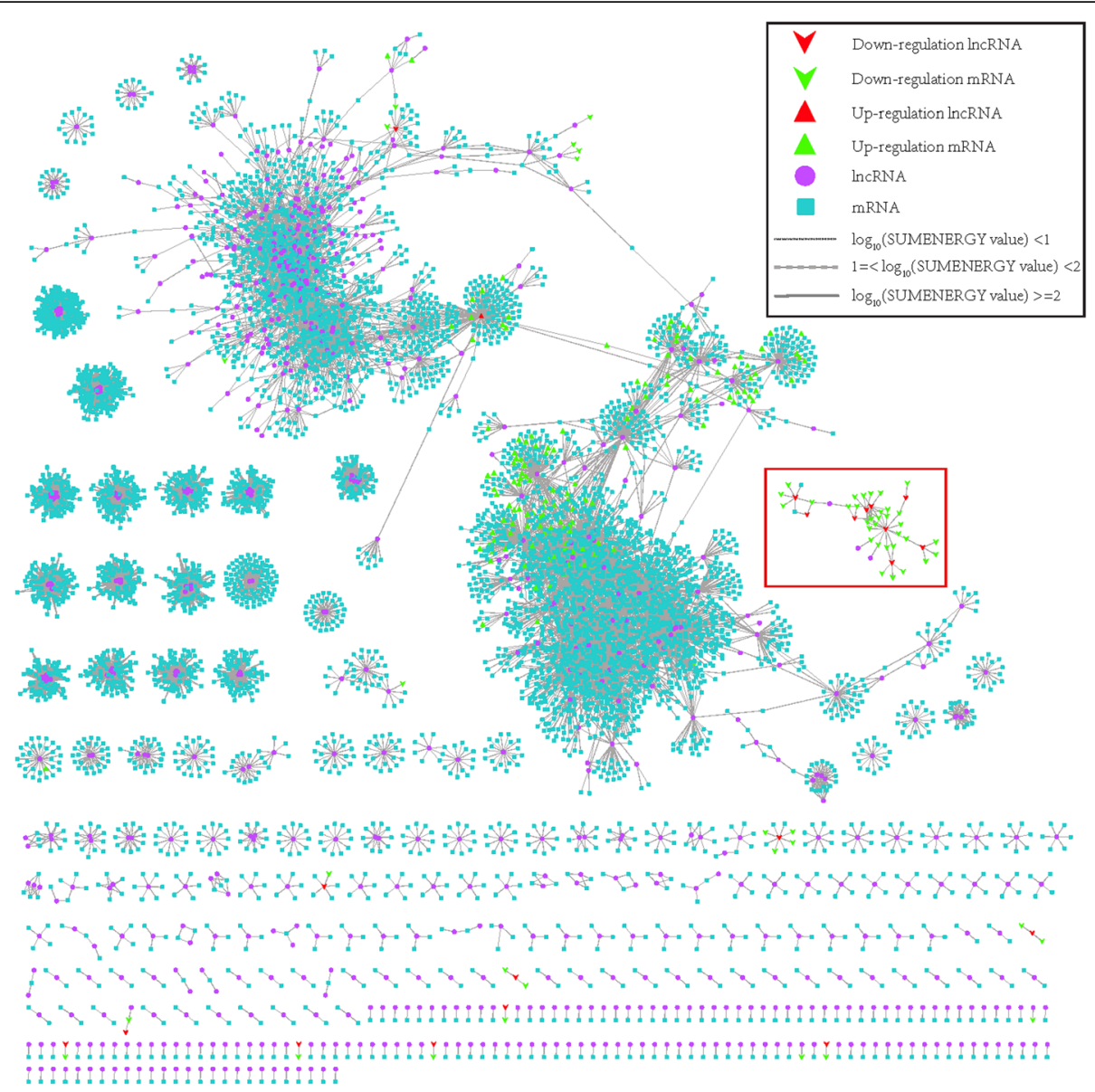

Fig. 6 mRNAs-IncRNAs interaction network constructed based on 23,784 mRNA-IncRNA pairs which involved in 815 IncRNAs and 6395 mRNAs. The free energy for hybridization evaluated by Rlblast program were used to weight the interaction edges. $\log 10($ SUMENERGY value) $<1$ were represented by thin lines, and $1=\log 10($ SUMENERGY value) $<2$ were represented by dotted lines, and $\log 10($ SUMENERGY value) $>=2$ were represented by thick lines

assembled for the subsequent analysis, of which 7814 transcripts were firstly identified in A. digitifera. These novel transcripts we found might contribute to novel changes of biological pathways and processes involved in Symbiodinium infection discovered in our study. In fact, symbiotic relationship between coral and Symbiodinium that could affect the cell differentiation and apoptosis has been observed in previous work on cnidarian-algal symbioses [48]. Dunn, SR et al. found that heat stress could induce programmed cell death (PCD) and necrosis in both host (sea anemone Aiptasia sp.) tissue and zooxanthellae, and the frequency of PCD would reach the peak when zooxanthellae started to escape from host during bleaching. Our findings were coherent with the conclusion of Dunn, SR et al. Differential expression analysis demonstrated several transcripts which were consistently up-regulated during $4 \mathrm{~h}$ post Symbiodinium infection were significantly enriched in GO_BP categories correlated to cell differentiation and apoptosis. Positive regulation of telomerase RNA localization to Cajal body appears to be related to activation of telomerase, which is an essential step in cellular immortalization [49]. P38 MAPKs are responsive to stress stimuli, such as heat shock (heat stress could induce bleaching in coral), and are involved not only in stress-induced signaling in cell differentiation, apoptosis and autophagy, but also in inflammatory response [50]. It is implied that initial establishment of symbiotic relationship between host coral and Symbiodinium, to a certain extent, could affect not only cell differentiation, apoptosis of host tissue, but also immune functions of host. In case of Pinzon J. H. et al's study [16], it was suggested that immune system appeared to be suppressed after bleaching. Based on our analysis, in addition to novel transcripts, we also identified some novel isoforms for the existed transcripts of RefTranscriptome_v1.0 (Fig. 3b). Furthermore, identification of alternative splicing modes among six statuses (control in $4 \mathrm{~h}, 12 \mathrm{~h}, 48 \mathrm{~h}$ and Symbiodinium-infection in $4 \mathrm{~h}, 12 \mathrm{~h}, 48 \mathrm{~h}$ ) indicated that the proportion of five main alternative splicing modes seemed not to be affected 
Table 2 Functional summary of target mRNAs of 21 differentially expressed IncRNAs in A. digitifera

\begin{tabular}{|c|c|c|c|}
\hline LncRNA ID & $\begin{array}{l}\text { Interaction } \\
\text { relationships }\end{array}$ & $\begin{array}{l}\text { Uniprot Annotation } \\
\text { of target mRNAs }\end{array}$ & $\begin{array}{l}\text { Functional analysis of } \\
\text { target mRNAs by David }\end{array}$ \\
\hline MSTRG.13003.1 & 129 & & $\begin{array}{l}\text { lung epithelial cell differentiation; protein kinase activity; } \\
\text { protein binding; Metabolic pathways; phosphorylation; } \\
\text { nucleobase-containing compound metabolic process; } \\
\text { cytoskeleton organization; GTP binding; positive } \\
\text { regulation of protein catabolic process; GTPase activity }\end{array}$ \\
\hline MSTRG.34897.1 & 19 & & protein K11-linked ubiquitination \\
\hline MSTRG.35238.1 & 16 & & $\begin{array}{l}\text { cytochrome-c oxidase activity; mitochondrion; } \\
\text { mitochondrial inner membrane; mitochondrial respiratory } \\
\text { chain complex IV; proton-transporting ATPase activity; } \\
\text { hydrogen ion transmembrane transport }\end{array}$ \\
\hline MSTRG.17668.1 & 10 & $\begin{array}{l}\text { ATP synthase subunit epsilon, mitochondrial; Maternal } \\
\text { protein exuperantia; COP9 signalosome complex subunit } \\
\text { 9; } 60 \mathrm{~S} \text { ribosomal protein L37a; } 40 \mathrm{~S} \text { ribosomal protein } \\
\text { S29;-;;;-; }\end{array}$ & \\
\hline MSTRG.14204.1 & 9 & $\begin{array}{l}\text { ATP synthase subunit epsilon, mitochondrial; 60S } \\
\text { ribosomal protein L37a; } 40 \mathrm{~S} \text { ribosomal protein S29; } \\
60 \mathrm{~S} \text { ribosomal protein L39-1;-; ; -; - }\end{array}$ & \\
\hline MSTRG.16403.1 & 8 & $\begin{array}{l}\text { PDZ and LIM domain protein Zasp; Golgi-associated plant } \\
\text { pathogenesis-related protein } 1 ; \text { WW domain-binding } \\
\text { protein 2; Outer dense fiber protein 1;;-;;;-; }\end{array}$ & \\
\hline MSTRG.39215.1 & 6 & 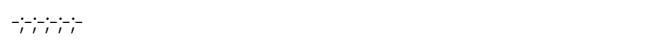 & \\
\hline MSTRG.34265.1 & 5 & $\begin{array}{l}\text { Collagen triple helix repeat-containing protein 1; } \\
\text { Uncharacterized protein YML079W; } \\
\text { Metallo-beta-lactamase domain-containing protein 2;-;- }\end{array}$ & \\
\hline MSTRG.6940.1 & 5 & & Ribosome; structural constituent of ribosome \\
\hline MSTRG.36289.1 & 4 & $\begin{array}{l}\text { V-type proton ATPase subunit e; } \\
\text { Chitobiosyldiphosphodolichol beta-mannosyltransferase; } \\
\text { Transcription initiation factor TFIID subunit 10;- }\end{array}$ & \\
\hline MSTRG.9806.1 & 3 & ATP synthase subunit epsilon, mitochondrial;-;- & \\
\hline MSTRG.19793.1 & 2 & $-;$ & \\
\hline MSTRG.33259.1 & 2 & Cilia- and flagella-associated protein 20; Protein FAM183B & \\
\hline MSTRG.3737.1 & 2 & Ubiquitin-like protein; c-Myc-binding protein & \\
\hline MSTRG.7448.1 & 2 & $-;$ Cytochrome $\mathrm{c}$ oxidase subunit 6B1 & \\
\hline MSTRG.13804.1 & 1 & - & \\
\hline MSTRG.21959.1 & 1 & - & \\
\hline MSTRG.29713.1 & 1 & 395 ribosomal protein L15, mitochondrial & \\
\hline MSTRG.32535.1 & 1 & Protein TAR1 & \\
\hline MSTRG.38024.1 & 1 & $60 S$ ribosomal protein $L 23$ & \\
\hline MSTRG.4196.1 & 1 & ATP synthase subunit delta, mitochondrial & \\
\hline
\end{tabular}

during Symbiodinium infection but could be affected as time goes on. For example, intron retention accounts for the largest proportion at $4 \mathrm{~h}$ time point (no matter in control or in Symbiodinium infection), nevertheless mutually exclusive exon dominated at $12 \mathrm{~h}$ and $48 \mathrm{~h}$ time points (Fig. 2d).

Compared to our previous lncRNAs study in coral [30], we applied a similar bioinformatic analysis pipeline. The major difference between two studies is that the whole genome, transcriptome as well as protein-coding gene dataset of $A$. digitifera has already been figured out [14], which allows us to obtain a high-confidence transcriptome dataset during the initial transcriptome assembly. In addition, protein-coding gene dataset could help us filter out more protein-coding RNAs from the initial assembled transcripts,resulting in a relatively high-confidence lncRNAs dataset. That may explain why we just predicted around 7000 lncRNAs for $A$. digitifera, whereas our previous study could obtain around 20,000 lncRNAs for two corals. Comparison of those lncRNAs between two studies showed that a small proportion of them exhibited sequence homologs, despite the close phylogenetic relationship. Furthermore, target mRNAs prediction of lncRNAs based on Pearson correlation 


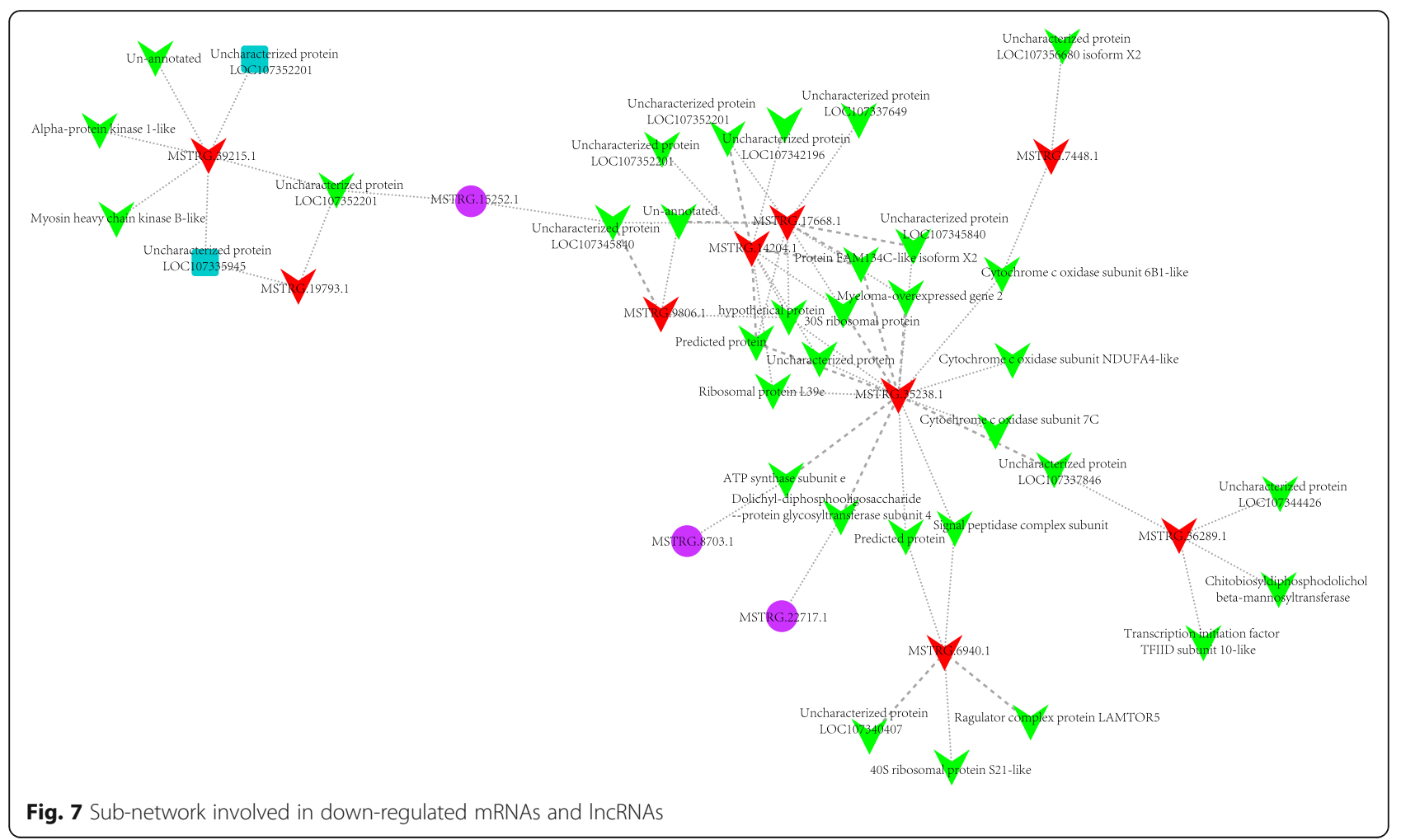

coefficient unmasked a complex regulatory network involved in numerous mRNAs-lncRNAs interactions exhibited in A. digitifera. More importantly, among those interactions, we noticed that a few lncRNAs were up/ down-regulated at initial Symbiodinium infection. For example, IncRNA MSTRG.13003.1 was up-regulated at post $04 \mathrm{~h}$ infection, and our network analysis showed that a total of 126 mRNAs significantly correlated to this lncRNA. Functional analysis achieved on DAVID website for those 126 co-expressed mRNAs demonstrated that the lncRNA MSTRG.13003.1 might regulate diverse biological processes and pathways involved in protein kinase activity, protein binding, metabolic pathways, phosphorylation cytoskeleton organization, GTP binding and positive regulation of protein catabolic process during the initial interaction with a competent strain of Symbiodinium (Table 2), implying that this lncRNA may play a crucial role for A. digitifera in response to Symbiodinium infection. For detailed functions and mechanism of these lncRNAs, further bench work and validations are required.

\section{Conclusions}

Based on our integrative transcriptome analysis, we detected numerous novel transcripts (including novel isoforms and alternative splicing modes) among six differential statuses of $A$. digitifera, as well as predicted a high-confidence dataset of lncRNAs for A. digitifera.
Besides, our analysis indicated that initial Symbiodinium infection not only affect the expression of mRNAs, but it could also induce ectopic expression of some specific lncRNAs, and functional investigation for these specific lncRNAs based on Pearson correlation coefficient demonstrated they correlated with levels of their putative target mRNAs involved in many biological processes and pathways at early stage of Symbiodinium infection, such as protein kinase activity, metabolic pathways, mitochondrion, ribosome, etc., and these target mRNAs were down-regulated, which implied the repression of these biological processes and pathways appeared to be tightly regulated via the specific lncRNAs. Our study provided a basis for the investigation of the molecular and cellular mechanism of noncoding RNAs involved in bleaching.

\section{Methods}

RNA sequence data derivation

As this study was conducted based on the previous published study [17], the raw RNA-seq data analyzed in the present study was downloaded from the NCBI Gene Expression Omnibus (GEO) database under Accession number GSE76976. For detailed information regarding the coral larvae and Symbiodinium culture, symbiont acquisition experimental design as well as larval sample and RNA sequencing, please refer directly to the Methods section at Mohamed A. R. et al's study [17]. 


\section{Data processing, reads alignment and assembly}

First, all the paired-end reads of $A$. digitifera larvae that were yielded based on Illumina HiSeq 2000 platform were filtered using Trimmomatic (version 0.36) [51] to remove adapter sequences and low-quality reads (Parameter: ILLUMINACLIP:TruSeq3-PE.fa:2:30:10:8:true SLIDINGWINDOW:4:15 LEADING:3 TRAILING:3 MINLEN:50). The statistics of raw data processing was achieved using in-house perl script. Then, the trimmed and clean-up reads were aligned to the $A$. digitifera genome assembly sequences v1.0 [14] (http://marinegen omics.oist.jp/genomes/) using HISAT2 (version 2.1.0) with default parameter [52]. The basic alignment statistics was calculated using samtools [53]. Afterwards, the bam files for each library obtained by alignment process were subjected to StringTie [54] to assemble into transcripts with default parameter. The final assembled transcripts were aligned to clean reads using Bowtie2 [55] to assess the transcriptome assembly quality, and Qualimap [31] was used to evaluate the alignment average coverage for the assembled transcripts.

\section{Quantification of RNA expression level and differential expression analysis}

The featureCounts (v1.5.3) [35] was initially used to quantify the abundances of the assembled transcripts for each library, and then the normalization of mapped reads counts as well as the differential expression analysis was done using the DESeq2 package [36] in the $\mathrm{R}$ statistical computing environment. Similar to the comparison strategy of Mohamed A. R. et al's study [17], the Symbiodinium-infected replicates were compared to the corresponding control replicates at $4 \mathrm{~h}, 12 \mathrm{~h}$ and $48 \mathrm{~h}$ time points, respectively. $P$-values for differential expression analysis were adjusted for multiple testing using Benjamini-Hochberg method, only the transcripts with a false discovery rate (FDR) of $\leq 0.05$ were retained.

\section{Functional annotation}

Initially, all the assembled transcripts were subjected to NCBI non-redundant (nr) protein database [56] and Swiss-Prot database [57] to search for the homologous sequences using BLASTx with cuf-off E-value of 1e-3. Then, GO and KEGG pathway enrichment analysis were performed on the specific datasets (i.e., differentially expressed RNAs, target mRNAs for differentially expressed lncRNAs) using the DATA FOR ANNOTATION, VISUALIZATION AND INTEGRATED DISCOVERY (DAVID) [37] (https://david.ncifcrf.gov/) and IPATH2 [58] (https://pathways.embl.de/). The UNIPROT accession identifiers of the top protein hits were extracted as identifiers for DAVID functional analysis and IPATH2 pathway analysis. The transcripts which were annotated to Swiss-Prot were served as the background for the enrichment analysis. Fisher's exact test were utilized to confirm statistically significant functional enrichment analysis, and GO categories and KEGG pathways with an EASE score (modified Fisher Exact $P$-Value) of $\leq 0.05$ were defined as significant [37].

\section{Identification of IncRNAs}

A stringent stepwise filtering pipeline was developed for identification of lncRNAs for A. digitifera (Fig. 1). The transcripts which were aligned to the protein sequences of $A$. digitifera [14] (v1.0) (http://marinegenomics.oist.jp/ genomes), NCBI non-redundant (nr) protein database as well as Swiss-Prot database were initially excluded. Then, the remaining transcripts with length less than $200 \mathrm{nt}$ were removed. Next, the remaining transcripts were translated (stop-to-stop codon) using in-house perl script, and only the longest ORF less than 100 amino acid residues (aa) was considered as putative lnRNA candidates. Furthermore, a tool called Coding Potential Calculator (CPC) [40] was utilized to assess the protein-coding potential of those putative lncRNA candidates. Lastly, the remaining transcripts that were predicted to encode any protein domains/motifs in Pfam database were filtered out using the localized version of Pfamscan [59].

\section{Functional investigation of IncRNAs}

To explore the function of lncRNAs of $A$. digitifera, we performed co-expression analysis between the predicted lncRNAs and mRNAs. For each of lncRNA, the Pearson Correlation Coefficient (PCC) of its expression value with that of each mRNA was first calculated using $\mathrm{R}$ package based on six different statuses (including $16 \mathrm{ex}-$ pression values for each lncRNA and mRNA). The IncRNA-mRNA pair that the absolute value of a PCC exceeded 0.95 and a $P$-value $<0.05$ was defined as co-expressed lncRNA-mRNA pair. To validate the significance of cutoff value determined as 0.95 , we proposed an individualized permutation test as follows: 100 lncRNAs was randomly selected from the lncRNAs dataset we had predicted, and then the 16 expression values for these selected 100 lncRNAs were randomly permutated. The mRNAs remained unchanged. After that, the PCC of the permutated expression values for the randomly selected 100 lncRNAs with that of each mRNA were re-calculated. These processes were repeated 1000 times. At last, we gathered all PCC values and calculated the proportion of $\mid$ Random $\mathrm{PCC} \mid \geq 0.95$. The result showed that the cutoff was set to 0.95 was sufficiently significant (the proportion $\mid$ Random PCC $\mid \geq 0.95=0.00004$ ).

On the other hand, we used RIblast [60] to compute the interaction probability for each possible IncRNA-mRNA pair based on free energy required for two RNA sequences hybridization. The less energy hybridization requires, the 
more probable two RNAs interact. The lncRNAs-mRNAs interaction network was visualized using Cytoscape (v3.51) [61]. For the specific IncRNAs set (i.e., hub lncRNAs, differentially expressed lncRNAs), their target mRNAs were subjected to the online annotation Tool DAVID to explore possible functions.

\section{Additional files}

Additional file 1: Table S1. Basic statistics of deep RNA sequencing data before and after processing. (DOCX $15 \mathrm{~kb}$ )

Additional file 2: Table S2. Basic statistics of clean reads alignment against to the coral A. digitifera genome sequence. (DOCX $14 \mathrm{~kb}$ )

Additional file 3: Table S3. Basic statistics of assembly results of transcriptome in A. digitifera. (DOCX $12 \mathrm{~kb}$ )

Additional file 4: Figure S1. Length distribution of all assembled traniscripts from A. digitifera transcriptome. (PDF $150 \mathrm{~kb}$ )

Additional file 5: Table S4. Basic statistics of clean reads alignment against to 59,904 assembled transcripts using Bowtie2. (DOCX $13 \mathrm{~kb}$ )

Additional file 6: Figure S2. Coverage distribution of 59,904 assembled transcripts evaluated based on all the clean reads. A) Coverage histogram of clean reads mapped into the assembled transcripts. B) Transcript fraction coverage of clean reads mapped into the assembled transcripts. (PDF $491 \mathrm{~kb}$ )

Additional file 7: Figure S3. Heatmap of whole transcriptome expression profiles. The heatmap was visualized using R package gplots, all expression values were normalized using Z-score using $R$ package DESeq2 and clustered based on "average" of hierarchical cluster analysis with R. (PDF $2619 \mathrm{~kb}$ )

Additional file 8: Figure S4. Comparison of expression value of all assembled traniscripts from $A$. digitifera transcriptome before and after normalization. (PDF $297 \mathrm{~kb}$ )

Additional file 9: Figure S5. Principle Component Analysis of expression value of all assembled traniscripts from $A$. digitifera transcriptome. (PDF $152 \mathrm{~kb}$ )

Additional file 10: File S1. GO and KEGG pathway analysis of 1036 differentially expressed transcripts achieved on David website. (XLSX 18 kb)

Additional file 11: Figure S6. Visualization of differentially expressed transcripts enriched in the metabolic pathways of. All differentially expressed mRNAs were subjected to the web-based tool IPath2.0 for visualization. Up-regulated genes are highlighted in red, down-regulated genes are highlighted in green. (PDF $2827 \mathrm{~kb}$ )

Additional file 12: File S2. GO and KEGG pathway analysis of target mRNAs for the IncRNAs involved in 23,784 mRNA-IncRNA interaction network achieved on David website. (XLSX 84 kb)

\section{Abbreviations}

aa: Amino acid residues; CPC: Coding Potential Calculator; DETs: Differentially expressed transcripts; FDR: False discovery rate; GEO: NCBI Gene Expression Omnibus database; GO: Gene Ontology; HST: High throughput sequencing; LncRNA: Long noncoding RNA; NGS: Next generation sequencing; $\mathrm{nr}$ database: NCBI non-redundant database; ORFs: Open reading frames; PCA: Principle component analysis; PCC: Pearson Correlation Coefficient; PCD: Programmed cell death; RefTranscriptome_v1.0: A. digitifera transcriptome assembly v1.0; UCR: Ultra-conserved region

\section{Acknowledgements}

Thanks to Yu Jin and Chang Chen for the suggestions of manuscript revision.

\section{Funding}

This work was supported by University of Macau through Research Grants SRG2016-00083-FHS, MYRG2018-00071-FHS, and FHS-CRDA-029-002-2017.

\section{Availability of data and materials}

All data used in this study is contained within the manuscript, in the additional files, or available for download from the NCBI Gene Expression Omnibus (GEO) database under Accession number GSE76976.

\section{Authors' contributions}

Research design and Bioinformatics analysis: $\mathrm{CH}$. Manuscript writing: $\mathrm{CH}$, XDZ, DLL, SXS. All authors read and approved the final manuscript.

Ethics approval and consent to participate

Not applicable.

\section{Consent for publication}

Not applicable.

\section{Competing interests}

The authors declare that they have no competing interests.

\section{Publisher's Note}

Springer Nature remains neutral with regard to jurisdictional claims in published maps and institutional affiliations.

Received: 19 January 2018 Accepted: 2 January 2019

Published online: 16 January 2019

\section{References}

1. Spalding M, Grenfell A. New estimates of global and regional coral reef areas. Coral Reefs. 1997;16(4):225-30.

2. Hoegh-Guldberg O, Mumby PJ, Hooten AJ, Steneck RS, Greenfield P, Gomez $E$, et al. Coral reefs under rapid climate change and ocean acidification. Science. 2007;318(5857):1737-42.

3. Pandolfi JM, Bradbury RH, Sala E, Hughes TP, Bjorndal KA, Cooke RG, et al. Global trajectories of the long-term decline of coral reef ecosystems. Science. 2003;301(5635):955-8.

4. Hughes TP, Baird AH, Bellwood DR, Card M, Connolly SR, Folke C, et al. Climate change, human impacts, and the resilience of coral reefs. Science. 2003:301(5635):929-33.

5. Lesser MP. Coral reef bleaching and global climate change: can corals survive the next century? Proc Natl Acad Sci U S A. 2007;104(13):5259-60.

6. Anthony KR, Kline DI, Diaz-Pulido G, Dove S, Hoegh-Guldberg O. Ocean acidification causes bleaching and productivity loss in coral reef builders. Proc Natl Acad Sci U S A. 2008;105(45):17442-6.

7. Donner SD, Knutson TR, Oppenheimer M. Model-based assessment of the role of human-induced climate change in the 2005 Caribbean coral bleaching event. Proc Natl Acad Sci U S A. 2007;104(13):5483-8.

8. Cheal AJ, MacNeil MA, Emslie MJ, Sweatman H. The threat to coral reefs from more intense cyclones under climate change. Glob Chang Biol. 2017; 23(4):1511-24.

9. Ainsworth TD, Heron SF, Ortiz JC, Mumby PJ, Grech A, Ogawa D, et al. Climate change disables coral bleaching protection on the great barrier reef. Science. 2016;352(6283):338-42.

10. Rowan R, Knowlton N, Baker A, Jara J. Landscape ecology of algal symbionts creates variation in episodes of coral bleaching. Nature. 1997;388(6639):265-9.

11. Warner ME, Fitt WK, Schmidt GW. Damage to photosystem II in symbiotic dinoflagellates: a determinant of coral bleaching. Proc Natl Acad Sci U S A. 1999;96(14):8007-12.

12. Roberts L. Coral bleaching threatens Atlantic reefs: unexplained changes are occurring in some of the most productive ecosystems on the planet, the Caribbean coral reefs. Science. 1987:238(4831):1228-9.

13. Meehan WJ, Ostrander GK. Coral bleaching: a potential biomarker of environmental stress. J Toxicol Environ Health. 1997;50(6):529-52.

14. Shinzato C, Shoguchi E, Kawashima T, Hamada M, Hisata K, Tanaka M, et al. Using the Acropora digitifera genome to understand coral responses to environmental change. Nature. 2011;476(7360):320-3.

15. DeSalvo MK, Voolstra CR, Sunagawa S, Schwarz JA, Stillman JH, Coffroth MA, et al. Differential gene expression during thermal stress and bleaching in the Caribbean coral Montastraea faveolata. Mol Ecol. 2008;17(17):3952-71.

16. Pinzon JH, Kamel B, Burge CA, Harvell CD, Medina M, Weil E, et al. Whole transcriptome analysis reveals changes in expression of immune-related genes during and after bleaching in a reef-building coral. R Soc Open Sci. 2015;2(4):140214. 
17. Mohamed AR, Cumbo V, Harii S, Shinzato C, Chan CX, Ragan MA, et al. The transcriptomic response of the coral Acropora digitifera to a competent Symbiodinium strain: the symbiosome as an arrested early phagosome. Mol Ecol. 2016;25(13):3127-41.

18. Perkel JM: Visiting "noncodarnia". In.; 2013.

19. Karapetyan AR, Buiting C, Kuiper RA, Coolen MW. Regulatory roles for long ncRNA and mRNA. Cancers. 2013;5(2):462-90.

20. Li H, Yu B, Li J, Su L, Yan M, Zhu Z, et al. Overexpression of IncRNA H19 enhances carcinogenesis and metastasis of gastric cancer. Oncotarget. 2014; 5(8):2318-29.

21. White NM, Zhao G, Zhang J, Davicioni E, Maher CA. Characterization of the novel IncRNA, PCAT14, clinically associated with metastatic prostate cancer: AACR; 2016. https://doi.org/10.1158/1538-7445.AM2016-974.

22. Li Z, Dong M, Fan D, Hou P, Li H, Liu L, Wu L, et al. LnCRNA ANCR downregulation promotes TGF- $\beta$-induced EMT and metastasis in breast cancer. Oncotarget. 2017;8(40):67329.

23. Nie W, Ge H-j, Yang X-q, Sun X, Huang H, Tao X, Chen W-s, Li B. LncRNAUCA1 exerts oncogenic functions in non-small cell lung cancer by targeting miR-193a-3p. Cancer Lett. 2016;371(1):99-106.

24. Mercer TR, Mattick JS. Structure and function of long noncoding RNAs in epigenetic regulation. Nat Struct Mol Biol. 2013;20(3):300-7.

25. Yang L, Froberg JE, Lee JT. Long noncoding RNAs: fresh perspectives into the RNA world. Trends Biochem Sci. 2014;39(1):35-43.

26. Zhao Y, Li H, Fang S, Kang Y, Wu W, Hao Y, et al. NONCODE 2016: an informative and valuable data source of long non-coding RNAs. Nucleic Acids Res. 2016;44(D1):D203-8.

27. Gaiti F, Fernandez-Valverde SL, Nakanishi N, Calcino AD, Yanai I, Tanurdzic $M$, et al. Dynamic and widespread IncRNA expression in a sponge and the origin of animal complexity. Mol Biol Evol. 2015;32(9):2367-82.

28. Ponting CP, Oliver PL, Reik W. Evolution and functions of long noncoding RNAs. Cell. 2009:136(4):629-41.

29. Quinn JJ, Chang HY. Unique features of long non-coding RNA biogenesis and function. Nat Rev Genet. 2016;17(1):47-62.

30. Huang C, Morlighem JRL, Cai J, Liao Q, Perez CD, Gomes PB, et al. Identification of long non-coding RNAs in two anthozoan species and their possible implications for coral bleaching. Sci Rep. 2017;7(1):5333.

31. Garcia-Alcalde F, Okonechnikov K, Carbonell J, Cruz LM, Gotz S, Tarazona S, et al. Qualimap: evaluating next-generation sequencing alignment data. Bioinformatics. 2012;28(20):2678-9.

32. Foissac S, Sammeth M. ASTALAVISTA: dynamic and flexible analysis of alternative splicing events in custom gene datasets. Nucleic Acids Res. 2007; 35(Web Server issue):W297-9.

33. Wang B, Tseng E, Regulski M, Clark TA, Hon T, Jiao Y, Lu Z, Olson A, Stein JC, Ware D. Unveiling the complexity of the maize transcriptome by singlemolecule long-read sequencing. Nat Commun. 2016;7:11708.

34. Wong JJ, Ritchie W, Ebner OA, Selbach M, Wong JW, Huang Y, et al. Orchestrated intron retention regulates normal granulocyte differentiation. Cell. 2013;154(3):583-95.

35. Liao Y, Smyth GK, Shi W. featureCounts: an efficient general purpose program for assigning sequence reads to genomic features. Bioinformatics. 2014;30(7):923-30.

36. Love Ml, Huber W, Anders S. Moderated estimation of fold change and dispersion for RNA-seq data with DESeq2. Genome Biol. 2014;15(12):550.

37. Huang d W, Sherman BT, Lempicki RA. Systematic and integrative analysis of large gene lists using DAVID bioinformatics resources. Nat Protoc. 2009; 4(1):44-57.

38. Rowan R. Coral bleaching: thermal adaptation in reef coral symbionts. Nature. 2004;430(7001):742.

39. Zvereva MI, Shcherbakova DM, Dontsova OA. Telomerase: structure, functions, and activity regulation. Biochemistry Biokhimiia. 2010;75(13):1563-83.

40. Kong L, Zhang Y, Ye ZQ, Liu XQ, Zhao SQ, Wei L, et al. CPC: assess the protein-coding potential of transcripts using sequence features and support vector machine. Nucleic Acids Res. 2007;35(Web Server issue):W345-9.

41. Chen CK, Yu CP, Li SC, Wu SM, Lu MJ, Chen YH, et al. Identification and evolutionary analysis of long non-coding RNAs in zebra finch. BMC Genomics. 2017;18(1):117.

42. Tsoi LC, lyer MK, Stuart PE, Swindell WR, Gudjonsson JE, Tejasvi T, et al. Analysis of long non-coding RNAs highlights tissue-specific expression patterns and epigenetic profiles in normal and psoriatic skin. Genome Biol. 2015;16:24.
43. Ren H, Wang G, Chen L, Jiang J, Liu L, Li N, et al. Genome-wide analysis of long non-coding RNAs at early stage of skin pigmentation in goats (Capra hircus). BMC Genomics. 2016;17:67.

44. Ulitsky I, Shkumatava A, Jan CH, Sive H, Bartel DP. Conserved function of lincRNAs in vertebrate embryonic development despite rapid sequence evolution. Cell. 2011;147(7):1537-50.

45. Chodroff RA, Goodstadt L, Sirey TM, Oliver PL, Davies KE, Green ED, et al. Long noncoding RNA genes: conservation of sequence and brain expression among diverse amniotes. Genome Biol. 2010;11(7):R72.

46. Johnsson P, Lipovich L, Grandér D, Morris KV. Evolutionary conservation of long non-coding RNAs; sequence, structure, function. Biochimica et Biophysica Acta (BBA)-General Subjects. 2014;1840(3):1063-71.

47. Huttemann M, Helling S, Sanderson TH, Sinkler C, Samavati L, Mahapatra G, et al. Regulation of mitochondrial respiration and apoptosis through cell signaling: cytochrome c oxidase and cytochrome c in ischemia/reperfusion injury and inflammation. Biochim Biophys Acta. 2012;1817(4):598-609.

48. Dunn S, Thomason J, Le Tissier M, Bythell J. Heat stress induces different forms of cell death in sea anemones and their endosymbiotic algae depending on temperature and duration. Cell Death Differ. 2004;11(11):1213-22.

49. Zhu Y, Tomlinson RL, Lukowiak AA, Terns RM, Terns MP. Telomerase RNA accumulates in Cajal bodies in human cancer cells. Mol Biol Cell. 2004;15(1):81-90.

50. Martin-Blanco E. p38 MAPK signalling cascades: ancient roles and new functions. BioEssays. 2000;22(7):637-45.

51. Bolger AM, Lohse M, Usadel B. Trimmomatic: a flexible trimmer for Illumina sequence data. Bioinformatics. 2014;30(15):2114-20.

52. Kim D, Langmead B, Salzberg SL. HISAT: a fast spliced aligner with low memory requirements. Nat Methods. 2015;12(4):357-60.

53. Li H, Handsaker B, Wysoker A, Fennell T, Ruan J, Homer N, Genome Project Data Processing $S$, et al. The sequence alignment/map format and SAMtools. Bioinformatics. 2009;25(16):2078-9.

54. Pertea M, Pertea GM, Antonescu CM, Chang TC, Mendell JT, Salzberg SL. StringTie enables improved reconstruction of a transcriptome from RNA-seq reads. Nat Biotechnol. 2015;33(3):290-5.

55. Langdon WB. Performance of genetic programming optimised Bowtie2 on genome comparison and analytic testing (GCAT) benchmarks. BioData mining. 2015;8(1):1.

56. Pruitt KD, Tatusova T, Maglott DR. NCBI reference sequence (RefSeq): a curated non-redundant sequence database of genomes, transcripts and proteins. Nucleic Acids Res. 2005;33(Database issue):D501-4.

57. The UniProt C. UniProt: the universal protein knowledgebase. Nucleic Acids Res. 2017;45(D1):D158-69.

58. Yamada T, Letunic I, Okuda S, Kanehisa M, Bork P. iPath2.0: interactive pathway explorer. Nucleic Acids Res. 2011;39(Web Server issue):W412-5.

59. Finn RD, Coggill P, Eberhardt RY, Eddy SR, Mistry J, Mitchell AL, et al. The Pfam protein families database: towards a more sustainable future. Nucleic Acids Res. 2016;44(D1):D279-85.

60. Fukunaga T, Hamada M. Rlblast: an ultrafast RNA-RNA interaction prediction system based on a seed-and-extension approach. Bioinformatics. 2017; 33(17):2666-74.

61. Shannon P, Markiel A, Ozier O, Baliga NS, Wang JT, Ramage D, et al. Cytoscape: a software environment for integrated models of biomolecular interaction networks. Genome Res. 2003;13(11):2498-504.

Ready to submit your research? Choose BMC and benefit from:

- fast, convenient online submission

- thorough peer review by experienced researchers in your field

- rapid publication on acceptance

- support for research data, including large and complex data types

- gold Open Access which fosters wider collaboration and increased citations

- maximum visibility for your research: over $100 \mathrm{M}$ website views per year

At $\mathrm{BMC}$, research is always in progress.

Learn more biomedcentral.com/submissions 